\title{
Neural-Network-Based Fuzzy Group Forecasting with Application to Foreign Exchange Rates Prediction
}

\author{
Lean $\mathrm{Yu}^{1,2}$, Kin Keung $\mathrm{Lai}^{2}$, and Shouyang Wang ${ }^{1}$ \\ ${ }^{1}$ Institute of Systems Science, Academy of Mathematics and Systems Science, \\ Chinese Academy of Sciences, Beijing 100080, China \\ \{yulean, sywang\} aamss.ac.cn \\ ${ }^{2}$ Department of Management Sciences, City University of Hong Kong, \\ Tat Chee Avenue, Kowloon, Hong Kong \\ \{msyulean, mskklai\} @cityu. edu.hk
}

\begin{abstract}
This study proposes a novel neural-network-based fuzzy group forecasting model for foreign exchange rates prediction. In the proposed model, some single neural network models are first used as predictors for foreign exchange rates prediction. Then these single prediction results produced by each single neural network models are fuzzified into some fuzzy prediction representations. Subsequently, these fuzzified prediction representations are aggregated into a fuzzy group consensus, i.e., aggregated fuzzy prediction representation. Finally, the aggregated prediction representation is defuzzified into a crisp value as the final prediction results. For illustration and testing purposes, a typical numerical example and three typical foreign exchange rates prediction experiments are presented. Experimental results reveal that the proposed model can significantly improve the prediction performance for foreign exchange rates.
\end{abstract}

Keywords: Artificial neural networks, fuzzy group forecasting, foreign exchange rates prediction.

\section{Introduction}

Foreign exchange rate forecasting has been a common research stream in the last few decades. Over this time, the research stream has gained momentum with the advancement of computer technologies, which have made many elaborate computation methods available and practical [1]. Due to its high volatility, foreign exchange rates forecasting is regarded as a rather challenging task. For traditional statistical methods, it is hard to capture the high volatility and nonlinear characteristics hidden in the foreign exchange market. As a result, many emerging artificial intelligent techniques, such as artificial neural networks (ANN), were widely used in foreign exchange rates forecasting and obtained good prediction performance. For example, De Matos [2] compared the strength of a multilayer feed-forward neural network (FNN) with that of a recurrent network based on the forecasting of Japanese yen futures. Kuan and Liu [3] provided a comparative evaluation of the performance of MLFN and a recurrent 
neural network (RNN) on the prediction of an array of commonly traded exchange rates. In the article of Tenti [4], the RNN is directly applied to exchange rates forecasting. Hsu et al. [5] developed a clustering neural network (CNN) model to predict the direction of movements in the USD/DEM exchange rate. Their experimental results suggested that their proposed model achieved better forecasting performance relative to other indicators. In a more recent study by Leung et al. [6], the forecasting accuracy of MLFN was compared with the general regression neural network (GRNN). The study showed that the GRNN possessed a greater forecasting strength relative to MLFN with respect to a variety of currency exchange rates. Similarly, Chen and Leung [7] adopted an error correction neural network (ECNN) model to predict foreign exchange rates. Yu et al. [8] proposed an adaptive smoothing neural network (ASNN) model by adaptively adjusting error signals to predict foreign exchange rates and obtained good performance.

However, neural networks are a kind of very unstable learning paradigm. Usually, small changes in the training set and/or parameter selection can produce large changes in the predicted output. To remedy the drawbacks, this paper attempts to utilize a set of neural network predictors to construct a fuzzy group forecasting methodology for foreign exchange rates prediction. In the proposed model, a number of single neural network models are first used as predictors for foreign exchange rates prediction. Then these single prediction results produced by each single neural network models are fuzzified into some fuzzy prediction representations. Subsequently, these fuzzified prediction representations are aggregated into a fuzzy group consensus, i.e., aggregated fuzzy prediction representation. Finally, the aggregated fuzzy prediction representation is defuzzified into a crisp value as the final prediction results.

The major aim of this study is to present a new forecasting paradigm called fuzzy group forecasting that can significantly improve the prediction capability of neural networks. The rest of this study is organized as follows. Section 2 describes the proposed neural network based fuzzy group forecasting model in detail. For further illustration, a typical numerical example and three typical foreign exchange rates prediction experiments are presented in Section 3. Section 4 concludes the study.

\section{Neural-Network-Based Fuzzy Group Forecasting Methodology}

In this section, a neural-network-based fuzzy group forecasting model is proposed for time series prediction problem. The basic idea of the fuzzy group forecasting model is to make full use of group member's knowledge to make a more accurate prediction over any single neural network predictors. For convenience of simplification, this study utilizes three group feed-forward neural network members to construct a fuzzy group forecasting model. Generally speaking, the neural-network-based fuzzy group forecasting consists of four different steps.

Step I: Single Neural Predictor Creation. In order to construct different single neural predictor for the neural network model with the same structure, we use different training sets to create different neural network predictors. In this study the bagging algorithm proposed by Breiman [9] is used to generate different training sets. 
Step II: Single Predictors Fuzzification. Based on these different training sets, each neural network can produce some different predictors. Using the different predictors, we can obtain different prediction results. Because neural predictor is an unstable learning paradigm, we are required to integrate these different results produced by different predictors, as earlier noted in Section 1. For these different predictions, we consider them as a fuzzy number for further processing. For example, suppose that the original dataset is used to create $k$ different training sets, i.e., $T R_{1}, T R_{2}, \ldots, T R_{k}$, via the bagging algorithm, we can use them to generate $k$ models, i.e., $f_{1}^{i}, f_{2}^{i}, \cdots f_{k}^{i}$, for the $i$ th neural network predictor. Accordingly, $k$ different predictions, i.e., $f_{1}^{i}(x), f_{2}^{i}(x), \cdots f_{k}^{i}(x)$, can be generated by the $i$ th neural network predictor when out-of-sample forecasting. In order to make full use of all information provided by different predictions, without loss of generalization, we utilize the triangular fuzzy number to construct the fuzzy representation for different predicted results. That is, the smallest, average and largest of the $k$ predictions are used as the left-, mediumand right membership degrees. That is, the smallest and largest values are seen as the optimistic and pessimistic prediction and the average value is considered to be the most likely prediction. Using this fuzzification method, one can make a fuzzy prediction judgment for each time series. More clearly, the triangular fuzzy number in this case can be represented as

$$
\begin{array}{r}
\tilde{Z}_{i}=\left(z_{i 1}, z_{i 2}, z_{i 3}\right)=\left(\left[\min \left(f_{1}^{i}(x), f_{2}^{i}(x), \cdots, f_{k}^{i}(x)\right)\right],\right. \\
\left.\left[\sum_{i=1}^{k} f_{j}^{i}(x) / k\right],\left[\max \left(f_{1}^{i}(x), f_{2}^{i}(x), \cdots, f_{k}^{i}(x)\right)\right]\right)
\end{array}
$$

In such a fuzzification processing way, the prediction problem is extended into a fuzzy group forecasting framework.

Step III: Fuzzy Prediction Aggregation. Suppose that there is $p$ different group members, i.e., $p$ different neural network predictors, let $\tilde{Z}=\psi\left(\tilde{Z}_{1}, \tilde{Z}_{2}, \cdots \tilde{Z}_{p}\right)$ be the aggregation of the $p$ fuzzy prediction representations where $\psi(\bullet)$ is an aggregation function. Now how to determine the aggregation function or how to aggregate these fuzzy prediction representations to be a group prediction consensus is an important and critical problem under fuzzy group forecasting environment. Generally speaking, there are many aggregation operators and rules that can be used to aggregate fuzzy prediction representations. Usually, the fuzzy prediction representations of $p$ group members will be aggregated using a commonly used linear additive procedure, i.e.,

$$
\tilde{Z}=\sum_{i=1}^{p} w_{i} \tilde{Z}_{i}=\left(\sum_{i=1}^{p} w_{i} z_{i 1}, \sum_{i=1}^{p} w_{i} z_{i 2}, \sum_{i=1}^{p} w_{i} z_{i 3}\right)
$$

where $w_{i}$ is the weight of the $i$ th group forecasting member, $i=1,2, \ldots, p$. The weights usually satisfy the following normalization condition:

$$
\sum_{i=1}^{p} w_{i}=1
$$

The key to this aggregation procedure is how to determine the optimal weight $w_{i}$ of the $i$ th fuzzy prediction representation under fuzzy group forecasting environment. 
Often the fuzzy representations of the predictions are largely dispersed and separated. In order to achieve the maximum similarity, the fuzzy prediction representations should move towards one another. This is the principle on the basis of which an aggregated fuzzy prediction representation is generated. Relied on this principle, a leastsquares aggregation optimization approach is proposed to integrate fuzzy prediction results produced by different prediction models.

The generic idea of this proposed aggregation optimization approach is to minimize the sum of squared distance from one fuzzy prediction to another and thus make them maximum agreement. Particularly, the squared distance between $\widetilde{Z}_{i}$ and $\widetilde{Z}_{j}$ can be defined by

$$
d_{i j}^{2}=\left(\sqrt{\left(w_{i} \tilde{Z}_{i}-w_{j} \tilde{Z}_{j}\right)^{2}}\right)^{2}=\sum_{l=1}^{3}\left(w_{i} z_{i l}-w_{j} z_{j l}\right)^{2}
$$

Using this definition, we can construct the following optimization model, which minimizes the sum of the squared distances between all pairs of weights fuzzy prediction representations:

$$
(P)\left\{\begin{array}{c}
\text { Minimize } D=\sum_{i=1}^{p} \sum_{j=1, j \neq i}^{p} d_{i j}^{2}=\sum_{i=1}^{p} \sum_{j=1, j \neq i}^{p}\left[\sum_{l=1}^{3}\left(w_{i} z_{i l}-w_{j} z_{j l}\right)^{2}\right] \\
\text { Subject to } \sum_{i=1}^{p} w_{i}=1 \\
w_{i} \geq 0, \quad i=1,2, \cdots p
\end{array}\right.
$$

In order to solve the above optimal weights, the constraint (7) is first not considered. If the solution turns out to be nonnegative, then constraint (7) is satisfied automatically. Using Lagrange multiplier method, Equations (5) and (6) can construct the following Lagrangian function:

$$
L(w, \lambda)=\sum_{i=1}^{p} \sum_{j=1, j \neq i}^{p}\left[\sum_{l=1}^{3}\left(w_{i} z_{i l}-w_{j} z_{j l}\right)^{2}\right]-2 \lambda\left(\sum_{i=1}^{p} w_{i}=1\right)
$$

Differentiating (8) with $w_{i}$, we can obtain

$$
\frac{\partial L}{\partial w_{i}}=2 \sum_{j=1, j \neq i}^{p}\left[\sum_{l=1}^{3}\left(w_{i} z_{i l}-w_{j} z_{j l}\right) z_{i l}\right]-2 \lambda=0 \text { for each } i=1,2, \ldots, p .
$$

Equation (9) can be simplified as

$$
(p-1)\left(\sum_{l=1}^{3} z_{i l}^{2}\right) w_{i}-\sum_{j=1, j \neq i}^{p}\left[\sum_{l=1}^{3}\left(z_{i l} z_{j l}\right)\right] w_{j}-\lambda=0 \text { for each } i=1,2, \ldots, p .
$$

Setting $W=\left(w_{1}, w_{2}, \ldots, w_{p}\right)^{T}, I=(1,1, \ldots, 1)^{T}$ and the $T$ denotes transpose, $b_{i j}=(p-1)\left(\sum_{l=1}^{3} z_{i l}^{2}\right), i=j=1,2, \cdots, p, b_{i j}=-\sum_{l=1}^{3}\left(z_{i l} z_{j l}\right), i, j=1,2, \cdots, p ; j \neq i$ and 


$$
B=\left(b_{i j}\right)_{p \times p}=\left[\begin{array}{cccc}
(p-1)\left(\sum_{l=1}^{3} z_{1 l}^{2}\right) & -\sum_{l=1}^{3}\left(z_{1 l} z_{2 l}\right) & \ldots & -\sum_{l=1}^{3}\left(z_{1 l} z_{p l}\right) \\
-\sum_{l=1}^{3}\left(z_{2 l} z_{1 l}\right) & (p-1)\left(\sum_{l=1}^{3} z_{2 l}^{2}\right) & \ldots & -\sum_{l=1}^{3}\left(z_{2 l} z_{p l}\right) \\
\ldots & \ldots & \ldots & \ldots \\
-\sum_{l=1}^{3}\left(z_{p l} z_{1 l}\right) & -\sum_{l=1}^{3}\left(z_{p l} z_{2 l}\right) & \ldots & (p-1)\left(\sum_{l=1}^{3} z_{p l}^{2}\right)
\end{array}\right]
$$

Using matrix form and above settings, Equations (10) and (6) can be rewritten as

$$
\begin{gathered}
B W-\lambda I=0 \\
I^{T} W=1
\end{gathered}
$$

Similarly, Equation (5) can be expressed in a matrix form as $D=W^{T} B W$. Because $D$ is a squared distance which is usually larger than zero, $B$ should be positive definite and invertible. Using Equations (12) and (13) together, we can obtain

$$
\begin{gathered}
\lambda^{*}=1 /\left(I^{T} B^{-1} I\right) \\
W^{*}=\left(B^{-1} I\right) /\left(I^{T} B^{-1} I\right)
\end{gathered}
$$

Since $B$ is a positive definite matrix, all its principal minors will be strictly positive and thus $B$ is a nonsingular $M$-matrix [10]. According to the properties of $M$-matrices, we know $B^{-1}$ is nonnegative. Therefore $W^{*} \geq 0$, which implies that the constraint in Equation (7) is satisfied.

Step IV: Aggregated Prediction Defuzzification. After completing aggregation, a fuzzy group consensus can be obtained by Equation (2). To obtain crisp value of credit score, we use a defuzzification procedure to obtain the crisp value for decisionmaking purpose. According to Bortolan and Degani [11], the defuzzified value of a triangular fuzzy number $\tilde{Z}=\left(z_{1}, z_{2}, z_{3}\right)$ can be determined by its centroid, which is computed by

$$
z=\frac{\int_{z_{1}}^{z_{3}} x \mu_{\tilde{z}}(x) d x}{\int_{z_{1}}^{z_{3}} \mu_{\tilde{z}}(x) d x}=\frac{\int_{z_{1}}^{z_{2}}\left(x \cdot \frac{x-z_{1}}{z_{2}-z_{1}}\right) d x+\int_{z_{2}}^{z_{3}}\left(x \cdot \frac{z_{3}-x}{z_{3}-z_{2}}\right) d x}{\int_{z_{1}}^{z_{2}}\left(\frac{x-z_{1}}{z_{2}-z_{1}}\right) d x+\int_{z_{2}}^{z_{3}}\left(\frac{z_{3}-x}{z_{3}-z_{2}}\right) d x}=\frac{\left(z_{1}+z_{2}+z_{3}\right)}{3}
$$

In this way, a final group forecasting consensus is computed with the above processes. For illustration and verification purposes, an illustrated numerical example and three typical foreign exchange rates are conducted. 


\section{Experiments}

In this section, we first present an illustrative numerical example to explain the implementation process of the proposed fuzzy group forecasting model using US dollar against Chinese Renminbi (USD/RMB) exchange rate series. Then three typical foreign exchange rates, US dollar against each of the three currencies - British pounds (GBP), euros (EUR) and Japanese yen (JPY), are used for testing. All four exchange data are obtained from Pacific Exchange Rates Services (http://fx.sauder.ubc.ca/), provided by Professor Werner Antweiler, University of British Columbia, Vancouver, Canada.

\subsection{An Illustrative Numerical Example}

Assume that there is USD/RMB series covered from January 1, 2006 to November 30, 2006, one would like to predict future USD/RMB exchange rate, e.g., December 1, 2006. For simplification, we first apply three standard FNN models with different topological structures to conduct this example. For example, we use three different numbers of hidden neurons to generate three different FNN models. In this example, we try to utilize five different models for prediction. For this purpose, the bagging sampling algorithm [9] is then used to create five different training sets. For each FNN model, five different training sets are used and five different prediction results are presented. With the above assumptions, three FNN models can produce 15 different prediction results, each for five predictions, as shown below.

FNN $(5-09-1)=(7.8211,7.8321,7.8451,7.8122,7.8247)$

FNN $(5-12-1)=(7.8309,7.8292,7.8302,7.8385,7.8278)$

FNN $(5-15-1)=(7.8082,7.8199,7.8208,7.8352,7.8393)$

where the numbers in parentheses indicate the topological structures of standard FNN model, for example, (5-12-1) represents five input nodes, twelve hidden nodes and one output node. Using Equation (1), the predictions of the three FNN models can be fuzzified into three triangular fuzzy numbers as fuzzy prediction representations, i.e.,

$$
\begin{aligned}
& \tilde{Z}_{F N N 1}=\left(z_{11}, z_{12}, z_{13}\right)=(7.8122,7.8270,7.8451) \\
& \tilde{Z}_{F N N 2}=\left(z_{21}, z_{22}, z_{23}\right)=(7.8278,7.8313,7.8385) \\
& \tilde{Z}_{F N N 3}=\left(z_{31}, z_{32}, z_{33}\right)=(7.8082,7.8247,7.8393)
\end{aligned}
$$

The subsequent task is to aggregate the three fuzzy prediction representations into a group prediction consensus. Using the above optimization method, we can obtain the following results:

$$
\begin{gathered}
B=\left[\begin{array}{ccc}
367.6760 & -183.9417 & -183.7432 \\
-183.9417 & 368.0916 & -183.8470 \\
-183.7432 & -183.8470 & 367.2971
\end{array}\right], \\
B^{-1}=10^{3} \times\left[\begin{array}{ccc}
2.1439 & 2.1427 & 2.1450 \\
2.1427 & 2.1415 & 2.1438 \\
2.1450 & 2.1438 & 2.1461
\end{array}\right],
\end{gathered}
$$




$$
W^{* T}=(0.3333,0.3332 ; 0.3335), \quad \tilde{Z}^{*}=\sum_{i=1}^{3} w^{*} \tilde{Z}_{i}=(7.8161,7.8277,7.8410)
$$

The final step is to defuzzify the aggregated fuzzy prediction value into a crisp prediction value. Using Equation (14), the defuzzified value of the final group prediction consensus is calculated by

$$
z=(7.8161+7.8277+7.8410) / 3=7.8282
$$

According to the data source, the actual value of USD/RMB in December 1, 2006 is 7.8283. By comparison, our fuzzy group prediction is rather promising. For further verification, three typical foreign exchange rates are tested.

\subsection{Three Foreign Exchange Rates Prediction Experiments}

In this subsection, three typical foreign exchange rates are used to test the effectiveness of the proposed neural network-based fuzzy group forecasting model. The data used here are monthly and they consist of the USD/GBP, USD/EUR and USD/JPY. We take monthly data from January 1971 to December 2000 as in-sample (training periods) data sets (360 observations including 60 samples for cross-validations). We also take the data from January 2001 to November 2006 as out-of-sample (testing periods) data sets (71 observations), which is used to evaluate the good or bad performance of prediction based on some evaluation measurement. For evaluation, two typical indicators, normalized mean squared error (NMSE) [1] and directional statistics $\left(D_{\text {stat }}\right)[1]$ are used. In addition, for comparison purpose, linear regression (LinR), logit regression $(\operatorname{LogR})$ and single FNN model are used here. Particularly, we use ten different FNN models with different structures to construct the fuzzy group forecasting model. In addition, the bagging algorithm [9] is used to create ten different training sets. Accordingly, the results obtained are reported in Table 1.

Table 1. The NMSE and $D_{\text {stat }}$ comparisons for different models

\begin{tabular}{lcccccc}
\hline \multirow{2}{*}{ Models } & \multicolumn{2}{c}{ GBP } & \multicolumn{2}{c}{ EUR } & \multicolumn{2}{c}{ JPY } \\
\cline { 2 - 7 } & $N M S E$ & $D_{\text {stat }}(\%)$ & $N M S E$ & $D_{\text {stat }}(\%)$ & $N M S E$ & $D_{\text {stat }}(\%)$ \\
\hline Single LinR Model & 0.0811 & 57.75 & 0.0892 & 59.15 & 0.2346 & 56.33 \\
Single LogR Model & 0.0792 & 63.38 & 0.0669 & 67.61 & 0.1433 & 70.42 \\
Single FNN Model & 0.0767 & 69.01 & 0.0663 & 70.42 & 0.1782 & 71.83 \\
Fuzzy Group Forecasting & 0.0083 & 84.51 & 0.0112 & 83.10 & 0.0458 & 81.69 \\
\hline
\end{tabular}

From Table 1, a clear comparison of various methods for the three currencies is given via NMSE and $D_{\text {stat }}$. Generally speaking, the results obtained from the two tables also indicate that the prediction performance of the proposed neural network based fuzzy group forecasting model is better than those of the single neural network model, linear regression and logit regression forecasting models for the three main currencies. The main reasons are that (1) aggregating multiple predictions into a group consensus can definitely improve the performance, as Yu et al. [1] revealed; (2) fuzzification of the predictions may generalize the model by processing some uncertainties of forecasting; and (3) as an "universal approximator", neural network might also make a contribution for the performance improvement. 


\section{Conclusions}

In this study, a new neural network based fuzzy group forecasting model is proposed for foreign exchange rates prediction. In terms of the empirical results, we can find that across different models for the test cases of three main currencies - British pounds (GBP), euros (EUR) and Japanese yen (JPY) — on the basis of different evaluation criteria, our proposed neural network based fuzzy group forecasting method performs the best. In the proposed neural network based fuzzy group forecasting cases, the NMSE is the lowest and the $D_{\text {stat }}$ is the highest, indicating that the proposed neural network based fuzzy group forecasting model can be used as a promising tool for foreign exchange rates prediction.

\section{Acknowledgements}

This work is supported by the grants from the National Natural Science Foundation of China (NSFC No. 70221001, 70601029), the Chinese Academy of Sciences (CAS No. 3547600), the Academy of Mathematics and Systems Sciences (AMSS No. 3543500) of CAS, and the Strategic Research Grant of City University of Hong Kong (SRG No. 7001677, 7001806).

\section{References}

1. Yu, L., Wang, S.Y., Lai, K.K.: A Novel Nonlinear Ensemble Forecasting Model Incorporating GLAR and ANN for Foreign Exchange Rates. Computers \& Operations Research 32 (2005) 2523-2541

2. De Matos G.: Neural Networks for Forecasting Exchange Rate. M. Sc. Thesis. The University of Manitoba, Canada (1994)

3. Kuan, C.M., Liu, T.: Forecasting Exchange Rates Using Feedforward and Recurrent Neural Networks. Journal of Applied Econometrics 10 (1995) 347-364

4. Tenti, P.: Forecasting Foreign Exchange Rates Using Recurrent Neural Networks. Applied Artificial Intelligence 10 (1996) 567-581

5. Hsu, W., Hsu, L.S., Tenorio, M.F.: A Neural Network Procedure for Selecting Predictive Indicators in Currency Trading. In Refenes A.N. (Ed.): Neural Networks in the Capital Markets. New York: John Wiley and Sons (1995) 245-257

6. Leung, M.T., Chen, A.S., Daouk, H.: Forecasting Exchange Rates Using General Regression Neural Networks. Computers \& Operations Research 27 (2000) 1093-1110

7. Chen, A.S., Leung, M.T.: Regression Neural Network for Error Correction in Foreign Exchange Rate Forecasting and Trading. Computers \& Operations Research 31 (2004) 1049-1068

8. Yu, L., Wang, S.Y., Lai, K.K. Adaptive Smoothing Neural Networks in Foreign Exchange Rate Forecasting. Lecture Notes in Computer Science 3516 (2005) 523-530

9. Breiman, L.: Bagging Predictors. Machine Learning 26 (1996) 123-140

10. Berman, A., Plemmons, R.J.: Nonnegative Matrices in the Mathematical Sciences. Academic, New York (1979)

11. Bortolan, G., Degani, R.: A Review of Some Methods for Ranking Fuzzy Subsets. Fuzzy Sets and Systems 15 (1985) 1-19 